\title{
INSTRUMENTO PARA A REALIZAÇÃO DE EXAME FÍSICO: CONTRIBUINDO PARA O ENSINO EM ENFERMAGEM
}

\author{
Tool for the performace of physical examination: contributing to nursing education \\ Instrumento para la realización de examen físico: contribuyendo para la enseñanza en \\ enfermería
}

Viviane Sales Freire Silva ${ }^{1}$

Dalmo Valério Machado de Lima²

Patrícia dos Santos Claro Fuly ${ }^{3}$

\begin{abstract}
RESUMO
Objetivou-se elaborar uma proposta de instrumento para a realização de exame físico de adultos e idosos por acadêmicos de enfermagem. 0 instrumento proposto derivou da análise obtida pela coleta de dados sobre exame físico por meio de revisão de literatura e de mapeamento cruzado com a Classificação Internacional para a Prática de Enfermagem CIPE ${ }^{\circledR}$ para a adição, subtração ou adequação com terminologia preexistente. 0 cotejamento entre as versões evidenciou que, de 296 termos descritos na literatura, apenas $66(22,1 \%)$ eram previstos na CIPE $^{\circledR}$. A versão final do instrumento proposto consta dos itens: nome, idade, leito, número de prontuário, diagnóstico médico, data de realização do exame, setor de internação, e itens sobre: nível de consciência e dependência, estado geral, cabeça e pescoço, tórax, órgãos genitais, membros e dispositivos invasivos, incluindo legendas norteadoras e uma legenda k relacionada às alterações de pele no final do instrumento. Conclui-se que a versão final está pronta para ser testada com os acadêmicos.
\end{abstract}

Palavras-chave: Exame físico. Processos de enfermagem. Ensino.

\begin{abstract}
The objective was to develop a proposal for an instrument to carry out physical examination of adults and elderly by nursing students. The proposed instrument was derived from analysis obtained by collecting data on physical examination by means of literature review and cross-mapping with the International Classification for Nursing Practice (CIPE) for addition, subtraction or fit with pre-existing terminology. The comparison between the versions showed that from 296 as described in the literature, only $66(22,1 \%)$ were provided in the CIPE. The final version of the proposed instrument consists of items: name, age, bed, number of records, medical diagnosis, date of examination, hospital sector, items: level of consciousness and dependency, general condition, head and neck, chest, genitals, limbs and invasive devices, including subtitles and a guiding legend $k$ related to skin changes at the end of the instrument. It is concluded that the final version is ready to be tested along with academics.
\end{abstract}

Keywords: Physical Examination. Nursing Process. Teaching.

\section{Resumen}

Se objetivó desarrollar una propuesta de instrumento para llevar a cabo el examen físico de adultos y ancianos por los estudiantes de enfermería. El instrumento propuesto se deriva del análisis obtenido por la recolección de datos sobre examen físico por medio de la revisión de la literatura y el mapeo cruzado con la Clasificación Internacional para la Práctica de Enfermería (CIPE ${ }^{\circledR}$ ) para sumar, disminuir o encajar con la terminología pre-existente. La comparación entre las versiones mostró que de 296 termos descriptos en la literatura, sólo $66(22,1 \%)$ se presentaron en la CIPE ${ }^{\circledR}$. La versión final del instrumento propuesto se compone de los elementos: nombre, edad, cama, número de registros, diagnóstico médico, la fecha del examen, sector hospitalario, y los ítems: el nivel de conciencia y de dependencia, estado general, cabeza y cuello, el pecho, órganos genitales, extremidades y dispositivos invasivos, incluyendo subtítulos y una leyenda $\mathrm{K}$ relacionada a los cambios de piel al final del instrumento. Se concluye que la versión final está lista para ser probada junto a los académicos.

Palabras clave: Examen Físico. Procesos de Enfermería. Enseñanza.

\footnotetext{
'Enfermeira Graduada em Licenciatura e Bacharelado pela Universidade Federal Fluminense. Residente do Instituto Estadual de Hematologia Arthur de Siqueira Cavalcanti pela Universidade Federal do Estado do Rio de Janeiro. (HEMORIO/UNIRIO) Rio de Janeiro - RJ. Brasil. E-mail: vivisafre@yahoo.com.br; Enfermeiro, Mestre e Doutor em Enfermagem, Professor Adjunto do Departamento de Enfermagem Médico-Cirúrgica da Escola de Enfermagem Aurora de Afonso Costa da Universidade Federal Fluminense, Grupo de Estudos de Sistematização da Assistência de Enfermagem (GESAE-UFF), Programa de Pós-Graduação Stricto-Sensu de Mestrado Profissional em Assistência de Enfermagem (MPEA-UFF), Programa de Pós-Graduação Stricto-Sensu em Ciências Cardiovasculares (PPGCCV-UFF). Niterói - RJ. Brasil. E-mail: dalmomachado@enf.uff.br; ${ }^{3}$ Doutora em enfermagem. Professora adjunta do departamento de enfermagem médico-cirúrgica da Escola de Enfermagem Aurora de Afonso Costa/Universidade Federal Fluminense. Niterói - RJ. Brasil. E-mail: patriciafuly@vm.uff.br
} 


\section{INTRODUÇÃO}

0 avanço tecnológico tem implicações em todas as áreas do conhecimento, entre elas a de saúde e, nela incluída, a enfermagem. Acredita-se que ele traz consigo repercussões positivas no campo teórico e prático, podendo proporcionar meios favoráveis de comunicação, inclusive a elaboração de instrumentos didáticos facilitadores à educação profissional.

Tecnologia pode ser concebida como conhecimento aplicado para a prevenção, diagnóstico e tratamento de doenças e reabilitação de suas consequências. Sendo assim, o saber fazer da enfermagem pode ser entendido como tecnologia ${ }^{1}$.

As tecnologias voltadas ao cuidado podem ser classificadas em: tecnologias duras, leve-duras e leves. As tecnologias leves correspondem a um momento de fala, de escuta e interpretações entre cuidador e o cliente; nas tecnologias duras, o profissional se utiliza de equipamentos tecnológicos para cuidar; e as tecnologias leve-duras respaldamse em um saber estruturado para fazê-lo, representado pelas disciplinas que operam em saúde, como a clínica médica, odontologia, epidemiologia, psicofarmacologia e outras².

Especificamente, a implementação do processo de enfermagem refere-se à tecnologia leve-dura, uma vez que envolve todo um sistema de conhecimentos científicos, que depende fundamentalmente de saberes entrelaçados, como a anatomia, fisiologia, farmacologia, microbiologia, entre outros; isso associado ao diálogo e à escuta sensível que estão presentes e definem a ação da enfermeira, possibilitando a construção de uma consciência crítica para os envolvidos no cuidado ${ }^{3}$.

Tem-se que a referida implementação promove a valorização profissional dos enfermeiros diante dos demais membros da equipe de enfermagem e do corpo de saúde, exigindo uma postura mais resolutiva e gerando aproximação dos pacientes, com consequente individualização do cuidado. Esses fatores contribuem para a qualidade da assistência ${ }^{4}$.

Atualmente, a utilização dessa tecnologia é regulamentada pela Resolução 358/2009 do Conselho Federal de Enfermagem do Brasil, que a tornou obrigatória/compulsória em todo território nacional e em todos os cenários onde se dá o exercício clínico pelo enfermeiro. A referida resolução aponta que é de competência desse profissional o registro formal das informações no prontuário sobre a execução do processo de enfermagem por meio de um resumo dos dados coletados, dos diagnósticos de enfermagem, das intervenções implementadas, bem como dos resultados alcançados ${ }^{5}$.

Diante desta demanda, reflete-se sobre a necessidade de criação de instrumentos ou uso de instrumentos que possam auxiliar o enfermeiro nas atividades relacionadas ao registro diário do citado resumo nos prontuários dos clientes. Esses registros ou anotações de enfermagem consistem na forma de comunicação escrita de informações pertinentes ao cliente e aos seus cuidados. Entende-se que os registros são elementos imprescindíveis no processo de cuidado humano, visto que, quando redigidos de maneira que retratam a realidade a ser documentada, possibilitam a comunicação permanente ${ }^{6}$.

A informática, por sua vez, constitui-se em uma ferramenta tecnológica, cuja aplicação vem crescendo acentuadamente na área de saúde, com repercussões em todas as subáreas, como na enfermagem. Isso porque o papel da informatização na enfermagem é ajudar o enfermeiro a organizar e administrar um montante de informações, fornecendo, todo e qualquer dado que necessita para o desenvolvimento de suas ações. A informação é, portanto, um componente crítico da tomada de decisão eficaz e da qualidade da prática de enfermagem?

Dada a importância da informação para a enfermagem, a informática constitui-se em um pilar da Prática Baseada em Evidências (PBE). Podemos resumir que a PBE se dirige a apoiar a experiência clínica com dados provenientes da epidemiologia clínica, complementadas com revisões sistemáticas da literatura, para critérios decisórios em condutas assistenciais. ${ }^{8}$

A aplicabilidade da PBE demonstra-se no apoio à tomada de decisões com vistas à resolução de problemas advindos do cotidiano do cuidar. Assim, faz-se necessário que as evidências sejam buscadas para sustentar as decisões clínicas de diagnóstico, intervenções e resultados. Na prática clínica de enfermagem, 0 ato diagnóstico vem pelas associações entre as manifestações apresentadas pelos clientes (dados objetivos e subjetivos) ${ }^{9}$.

Vale ressaltar que não basta somente a identificação de problemas, uma vez que esses podem ser reportados de diferentes maneiras, a julgar pelo examinador que, dependendo de sua cultura, linguagem, formação acadêmica dentre outros fatores, pode ser influenciado no momento dos registros de enfermagem. Para realização de uma avaliação criteriosa do cliente, com implicação futura na formação dos diagnósticos e intervenções de enfermagem, julga-se necessária a incorporação de sistemas de classificação da prática de enfermagem, para que a comunicação entre os enfermeiros ocorra de forma mais efetiva ${ }^{10}$.

A criação de um sistema mais completo de classificação foi necessária e teve seu desenvolvimento iniciado em 1989, pelo Conselho Internacional de Enfermagem (CIE). Desde então, - CIE vem estimulando o desenvolvimento e adoção da Classificação Internacional para a Prática de Enfermagem (CIPE), que enfatiza a necessidade de uma abordagem unificadora, promovendo a integração e harmonização de múltiplas terminologias de enfermagem entre países e idiomas ${ }^{10}$.

Por meio da CIPE, acredita-se que seja possível impulsionar a padronização da linguagem de enfermagem e facilitar a avaliação da qualidade da assistência, considerando a sistematização, registro e quantificação do que os componentes da equipe de enfermagem produzem e, 
consequentemente, identificando a real participação e contribuição da enfermagem no cuidado à saúde das pessoas ${ }^{11}$.

Sendo assim, vale ressaltar que a utilização da CIPE poderá servir para tornar a prática de enfermagem visível nos sistemas de informação da saúde, a fim de que pesquisadores, educadores e gestores possam, a partir desses dados, identificar a contribuição da enfermagem no cuidado à saúde da clientela ${ }^{11}$.

Para tanto, considera-se importante que enfermeiros desenvolvam o pensamento crítico e sua capacidade de tomar decisões, já que são atuantes diretos no processo saúdedoença e no bem-estar dos indivíduos, famílias e comunidade, compreendendo que o objeto da enfermagem é o cuidado humano e que este deve ser prestado com qualidade e ser realizado com planejamento e de forma sistematizada, o que pode ocorrer por meio da implementação do processo de enfermagem ${ }^{12}$.

A identificação dos problemas apresentados pelo cliente e obtidos principalmente durante a realização do histórico de enfermagem, quando o enfermeiro entrevista, observa e realiza o exame físico, constitui um momento essencial à implementação do processo de enfermagem e que subsidiará a realização de todas as outras etapas posteriores.

0 processo de enfermagem tem representado 0 principal modelo metodológico para o desempenho sistemático da prática profissional, ou um instrumento tecnológico de que se lança mão para favorecer o cuidado, para organizar as condições necessárias à realização do cuidado e para documentar a prática profissional. A conceituação e a aplicação do processo de enfermagem evoluíram para o reconhecimento de que, além daquele julgamento estritamente vinculado à doença e suas complicações, os agentes da enfermagem fazem outros tipos de julgamentos e agem com base nestes. A aplicação deliberada e sistemática do processo de enfermagem pode acrescentar qualidade ao cuidado, melhorar a visibilidade e o reconhecimento profissional e representar uma possibilidade concreta de avaliação da prática profissional13.

0 exame físico precedido pela anamnese compõe a fase do histórico de enfermagem descrito anteriormente. Refere-se a uma revisão cefalocaudal do corpo humano para a investigação de cada sistema corporal do cliente, por meio da inspeção, da palpação, da percussão, da ausculta, do olfato e do uso de alguns instrumentos e aparelhos simples, fornecendo informações objetivas sobre ele e permitindo que o enfermeiro faça julgamentos clínicos ${ }^{14}$.

A execução do exame físico, em geral, representa o primeiro momento de contato físico com o paciente. As preocupações do aluno/enfermeiro em relação a sua competência para a realização dessa prática, bem como a manifestação de sentimentos como medo, ansiedade e insegurança, podem interferir diretamente na sua execução, ocasionando frustrações e receios diante do paciente. Por essa razão, tais questões devem ser consideradas ${ }^{15}$.

0 histórico de enfermagem pode ser traduzido como um roteiro sistematizado para o levantamento de dados do ser humano que são significativos para o enfermeiro e que tornam possível a identificação de seus problemas. Esses dados, convenientemente analisados e avaliados conduzem ao diagnóstico de enfermagem, que pode ser considerado como a identificação das necessidades humanas afetadas e a determinação pelo enfermeiro do grau de dependência para atendimento dessas necessidades, considerando sua natureza e extensão ${ }^{16}$.

Diante do diagnóstico estabelecido, ocorre 0 planejamento da assistência de enfermagem, quando são determinados os cuidados que o ser humano deverá receber. Após a confecção desse plano assistencial ocorre sua implementação, com base no roteiro diário. Esse se refere ao registro das mudanças sucessivas que ocorrem no ser humano enquanto está sob assistência profissional ${ }^{16}$.

Desse modo, o plano assistencial coordena a ação da equipe de enfermagem na execução dos cuidados adequados ao atendimento das necessidades básicas e específicas do cliente. 0 plano assistencial deve ser reavaliado sempre, fornecendo os dados necessários para a mensuração dos resultados de enfermagem. Pelos registros diários da enfermagem é possível avaliar a resposta do cliente à assistência de enfermagem implementada, julgando-se adequada ou não para a melhoria do seu quadro de saúde ${ }^{16}$.

Diante das questões apresentadas, a motivação para a construção de uma estratégia facilitadora do exame físico para utilização em situação de ensino de graduação em enfermagem surgiu a partir da prática docente e assistencial do(s) autor(es) do presente estudo, especificamente durante a experiência na clínica médica de um hospital universitário do estado do Rio de Janeiro, Brasil, com os acadêmicos de enfermagem do $5^{\circ}$ período da Escola de Enfermagem Aurora de Afonso Conta da Universidade Federal Fluminense. Nesse espaço institucional é desenvolvido o projeto de ensino "A arte de Ensinar/Aprender a cuidar de clientes adultos e idosos hospitalizados com doenças crônicas não transmissíveis", que desenvolve especificamente atividades relacionadas aos cuidados integrais a clientes.

Com base nas demandas do referido projeto, esse estudo tem por finalidade apresentar o instrumento a ser testado no processo de ensino e aprendizagem de alunos de graduação em enfermagem sobre exame físico de adultos e idosos hospitalizados, sendo traçado como objetivo: elaborar e apresentar uma proposta de instrumento para a realização da referida prática de enfermagem, a partir de revisão de literatura científica nacional e atual sobre o tema, cotejando a versão obtida com a CIPE. 


\section{MÉTODO}

Trata-se de uma revisão crítica da produção científica nacional e internacional de enfermagem, publicada sob forma de livros que contemplam 0 assunto foco desse estudo. A primeira versão do instrumento derivou da coleta de dados sobre o exame físico a partir da revisão de livros clássicos encontrados na biblioteca da Escola de Enfermagem Aurora de Afonso Costa entre agosto e setembro de 2009. Tais livros são demonstrados no Quadro 1, a seguir.

Quadro 1 - Livros clássicos contemplados na revisão de literatura.

\begin{tabular}{|l|l|l|}
\hline \multicolumn{1}{|c|}{ Título } & \multicolumn{1}{|c|}{ Autores } & \multicolumn{1}{|c|}{ Ano } \\
\hline Semiologia Médica & Celmo Celeno Porto & 2001 \\
\hline $\begin{array}{l}\text { Fundamentos de enfermagem, conceitos, processo e } \\
\text { prática. }\end{array}$ & $\begin{array}{l}\text { Patrícia Potter e Anne Griffin } \\
\text { Perry }\end{array}$ & 2005 \\
\hline $\begin{array}{l}\text { Tratado de enfermagem médico cirúrgico } \\
\text { Anamnese e exame físico: avaliação diagnóstica de } \\
\text { enfermagem no adulto }\end{array}$ & Alba Lúcia Barros \& Cols & 2005 \\
\hline
\end{tabular}

Procurou-se, nessa revisão, termos científicos referentes às possíveis alterações encontradas no corpo humano, além de sua divisão anatômica. Exemplos: Cabeça e pescoço; tórax; abdome; órgãos genitais; membros inferiores e membros superiores. A seguir, foram identificados esses termos científicos relacionando-os a cada parte do corpo. Exemplos: cabeça: couro cabeludo -lesão; cabeça: boca - mucosite; membros inferiores: pele-cianose

Após essa primeira etapa, seguiu-se a análise criteriosa dos termos obtidos, cotejando-os com aqueles existentes na CIPE ${ }^{\circledR}$ Versão 1 (CIE, 2005). Dessa forma, realizou-se um mapeamento cruzado da versão do instrumento que foi elaborado com base na revisão de literatura, com a CIPE para a adição, subtração ou adequação com terminologia preexistente, o que viabilizou a construção da segunda versão do instrumento. Durante o mapeamento, termos que foram incluídos na primeira versão do instrumento, como prótese dentária e dreno torácico, na segunda versão foram substituídos respectivamente por dentadura e tubo torácico. Outros exemplos de adequação das terminologias preexistentes são: comatoso derivando de coma; orientaçãoadequado para orientado; sonolência adequado para sonolento.

Assim, a segunda versão foi construída sob a forma de lista de múltipla escolha (checklist) e constitui a versão final proposta nesta oportunidade, que deverá ser testada em campo futuramente (Apêndices l e II).

\section{RESULTADOS E DISCUSSÃO}

Após a revisão crítica da literatura revisada e de seu cotejamento com a CIPE ${ }^{\circledR}$ Versão 1, a versão final do instrumento, de modo geral, consta de folha única para melhor manuseio pelos acadêmicos de enfermagem nas clínicas médicas em cardiologia, endocrinologia, hematologia, pneumologia e gastroenterologia. Ademais, a utilização de todo o espaço da folha, incluindo seu verso, poderá proporcionar melhor análise dos dados coletados do cliente para posterior formulação de um diagnóstico de enfermagem e, respectivamente, para a intervenção de enfermagem. A versão final do instrumento obtido foi dividida em quatro partes, para fins de melhor organização do espaço, e consta de:

Especificamente, na parte anterior da folha (Apêndice I) verificam-se: - cabeçalho: nome, idade, leito, prontuário, diagnóstico médico, data de realização do exame físico e setor de internação; - corpo do instrumento (divisão dos aspectos observados no exame físico organizado por letras): A) nível de consciência, B) dependência, C) estado geral, D) cabeça e pescoço, E) tórax subdividido em E.1) respiratório e E.2) cardíaco. Na parte posterior da folha (Apêndice II) verificamse: - corpo do instrumento (continuidade do exame físico): E.2) cardíaco, F) abdome, G) órgãos genitais, H) membros superiores, I) membros inferiores, J) dispositivos invasivos. Vale ressaltar que no Tópico I construiu-se uma tabela destacando o tempo e os sinais flogísticos observados ou não em cada dispositivo invasivo correspondente. Rodapé: legenda nomeada $\mathrm{K}$ sobre as diversas alteraç̧ões de pele. Além disso, no final do instrumento, julgou-se necessária a permanência de um espaço para possíveis observações imprescindíveis para a adequada avaliação do cliente analisados pelo enfermeiro responsável.

Em todo o corpo do instrumento foram criados quadros secundários para a caracterização de sinais e sintomas como: dor, massas e linfonodos palpáveis, segundo as respectivas localizações por regiões anatômicas. No segmento referente à dor, no que tange à avaliação da intensidade, propõe-se autilização de uma escala de quatro cruzes, onde uma cruz equivale a dor mínima e quatro cruzes equivale a dor máxima. Essa mesma escala é proposta para ser utilizada também na identificação da amplitude dos pulsos e da intensidade dos sopros.

Cabe salientar que a comparação entre a versão inicial, elaborada somente com base na literatura revisada, e a versão final, elaborada com o cotejamento proposto pela CIPE ${ }^{\circledR}$ Versão 1 evidenciou que, de 296 termos descritos na literatura, apenas 66 (22,1\%) eram previstos na CIPE ${ }^{\circledR}$ Versão 1. 
Para a realização do cotejamento entre as duas versões houve a necessidade de conhecer termo por termo para que fosse possível analisá-los quanto a sua origem e a sua disponibilidade, tanto na literatura revisada quanto na CIPE ${ }^{\circledR}$ Versão 1. Vale ressaltar que, após o cotejamento, foi dada a preferência aos termos que constam naCIPE ${ }^{\circledR}$ Versão 1, os quais foram 09 termos substituídos, 13 termos adequados e 48 termos não adequados, totalizando 70 termos previstos na CIPE ${ }^{\circledR}$ Versão 1. Porém observam-se entre eles 4 termos repetidos: normal; dor; audição; perfusão tissular.

OQuadro 2 demonstra os termos que tiveram que passar por adequaç̃̃es/substituições, bem como os que não tiveram, para se adequar ao instrumento.

Quadro 2 - Termos da versão final do instrumento de acordo com o cotejamento entre a literatura revisada e CIPE ${ }^{\circledR}$ Versão 1, classificando os termos que não tiveram necessidade de adequação e os que tiveram. Rio de Janeiro. 2010

\begin{tabular}{|c|c|c|}
\hline Termos sem adequação & \multicolumn{2}{|c|}{ Termos com adequação } \\
\hline LITERATURA-CIPE & LTERARURA & CIPE \\
\hline Consciência & Sondento & Sonolência/Sono \\
\hline Estado de Dependência & Orientado & Orientação \\
\hline Independente & Comatoso & Coma \\
\hline Parcial & Íntegro & Integridade \\
\hline Total & Doloroso & Dor * \\
\hline Regular & Desidratada & Desidratação \\
\hline Cabeça & Lateral & Lateralidade \\
\hline Normal * & Aumentado & Aumentar* \\
\hline Anormal & Dispneica & Dispneia \\
\hline Pescoço & Diminuído & Diminuir \\
\hline Face & Arrítmico & Arritmia \\
\hline Posterior & Ascítico & Ascite \\
\hline Anterior & Eritematosa & Eritema \\
\hline \multicolumn{3}{|l|}{ Antebraço } \\
\hline Couro cabeludo & \multicolumn{2}{|c|}{ Termos substituídos } \\
\hline Olhos & LITERATURA & CIPE \\
\hline Lesão & Boa & Normal * \\
\hline Prurido & Prótese dentária & Dentadura \\
\hline Visão & Sinais flogísticos & Sinais de Infecção \\
\hline Comprometido & Padrão Venoso & Perfusão Tissular* \\
\hline Nariz & Dreno torácico & Tubo torácico \\
\hline Obstrução & Sujidade auditiva & Cerúmen \\
\hline Ouvido & Sudorese & Úmida \\
\hline Dor* & Lábios cianóticos & Perfusão tissular em lábios \\
\hline Audição* & Hipoacusia/Surdez & Audição Comprometida* \\
\hline Língua & & NÃO CONSTA NA QPE \\
\hline Tórax & Hepatomegalia & Fígado $^{* *}$ aumentar $*$ \\
\hline Pele & Esplenomegalia & Baço $^{* *}$ aumentar * \\
\hline \multicolumn{3}{|l|}{ Mamas } \\
\hline \multicolumn{3}{|l|}{ Mamilo } \\
\hline \multicolumn{3}{|l|}{ Ritmo } \\
\hline \multicolumn{3}{|l|}{ Pulmão } \\
\hline \multicolumn{3}{|l|}{ Ruído } \\
\hline \multicolumn{3}{|l|}{ Região Vulvar } \\
\hline \multicolumn{3}{|l|}{ Testículo } \\
\hline \multicolumn{3}{|l|}{ Braço } \\
\hline \multicolumn{3}{|l|}{ Coxa } \\
\hline \multicolumn{3}{|l|}{ Perna } \\
\hline \multicolumn{3}{|l|}{ Movimento } \\
\hline \multicolumn{3}{|l|}{ Edema } \\
\hline \multicolumn{3}{|l|}{ Úlcera } \\
\hline \multicolumn{3}{|l|}{ Erosão } \\
\hline \multicolumn{3}{|l|}{ Fissura } \\
\hline \multicolumn{3}{|l|}{ Hematoma } \\
\hline \multicolumn{3}{|l|}{ Traqueostomia } \\
\hline \multicolumn{3}{|l|}{ lleostomia } \\
\hline \multicolumn{3}{|l|}{ Colostomia } \\
\hline Periférico & & \\
\hline
\end{tabular}

* Termos repetidos, porém contados apenas uma vez.

** Termos não encontrados na CIPE ${ }^{\circledR}$ Versão 1 referentes a termos científicos também não encontrados na CIPE ${ }^{\circledR}$ Versão 1. 
Para melhor análise quantitativa destes termos, apresenta-se o Gráfico 1.

Gráfico 1 - Frequência dos termos utilizados na versão final do instrumento conforme situação em relação ao cotejamento entre literatura revisada e a CIPE ${ }^{\circledR}$ Versão 1. Rio de Janeiro. 2010.

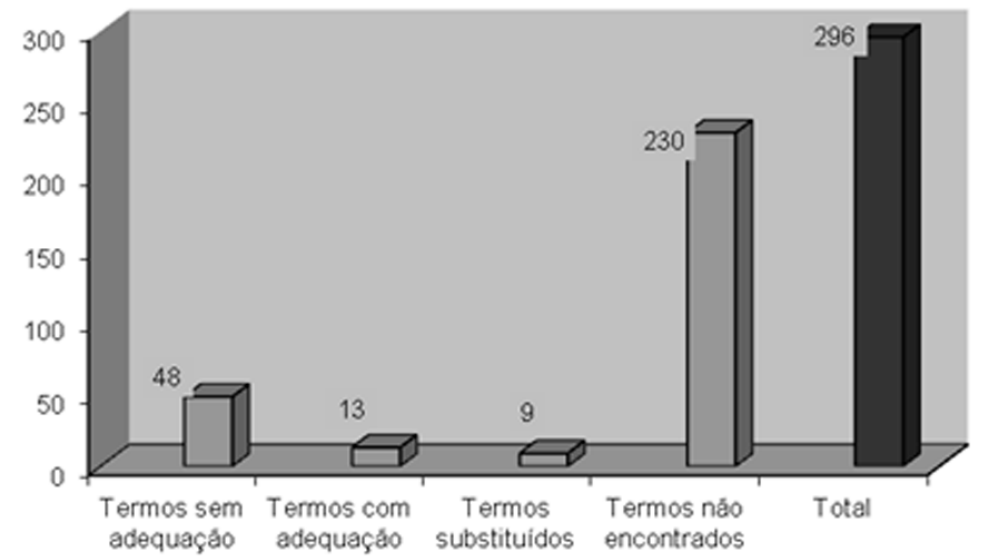

Esses dados demonstram a grande necessidade de se buscar novas pesquisas relacionadas à padronização dos sistemas de classificação em enfermagem, o que implicaria um avanço para a prática assistencial da enfermagem, principalmente em relação para favorecer a comunicação uniformizada entre os profissionais de saúde.

Pesquisas que identifiquem termos próprios da prática de enfermagem no Brasil ainda são escassas. Tal fato aponta para uma lacuna a ser preenchida no âmbito nacional, com 0 objetivo de agregar a $\mathrm{CIPE}{ }^{\circledR}$ à realidade da enfermagem brasileira, principalmente em seu contexto hospitalar, dado que a grande contribuição brasileira nessaárea está na Classificação Internacional para a Prática de Enfermagem em Saúde Coletiva - CIPESC, que abarca os termos relativos ao trabalho da enfermagem no âmbito da saúde pública no Brasil1" .

Além disso, proporcionará favorecimento no ensinoaprendizagem dos acadêmicos de enfermagem no que diz respeito ao conhecimento de várias terminologias existentes, bem como a sua importância para a sistematização da assistência de enfermagem de forma padronizada. Isso porque a existência de vários termos científicos com o mesmo significado dificulta a memorização e, consequentemente, promove um déficit no aprendizado.

Embora se deva afirmar que a utilização dos diferentes sistemas de classificação de linguagem da enfermagem que estão sendo construídos em âmbito mundial, a exemplo da CIPE ${ }^{\circledR}$, ainda é embrionária no Brasil, acredita-se que o uso dessas terminologias permita a identificação de padrões de cuidados que podem ser utilizados por enfermeiros em qualquer parte do mundo ${ }^{11}$.

Ainda que no Brasil o emprego dessas terminologias não seja obrigatório, cabe considerar a necessidade de discussão sobre elas e de sua implementação, com fins de crescimento e sustentação de uma prática profissional pautada no raciocínio clínico e crítico, bem como para acompanhar movimentos internacionais de padronização da linguagem de enfermagem ${ }^{17}$.

0 estudo pretendeu desenvolver um instrumento que facilita a realização do exame físico em adultos e idosos pelos alunos de graduação em enfermagem. Até a formação da versão final, houve inúmeros ajustes de acordo com os objetivos propostos pela pesquisa. 0 instrumento foi elaborado de forma a contemplar todos os sistemas e regiões anatômicas do corpo com suas possíveis alterações referentes a quadros patológicos.

Vale ressaltar que o instrumento está sendo testado juntamente com os acadêmicos de enfermagem e seus resultados serão posteriormente publicados. Acredita-se que tal instrumento pode estimular o interesse na aprendizagem e reconhecimento dos termos mapeados na CIPE ${ }^{\circledR}$. A partir de então, proporcionaria um registro de enfermagem padronizado e com qualidade, dado que as informações relativas ao exame físico poderiam ser detalhadas e completas, de modo que a assistência de enfermagemfosse baseada em evidências descritas e localizáveis nos registros de enfermagem pertencentes ao prontuário do cliente.

É importante que tanto os enfermeiros quanto os futuros enfermeiros e acadêmicos de enfermagem compreendam como a informatização pode modificar para melhor o seu trabalho diário. Os recursos computacionais são desenvolvidos para incrementar a produtividade e a qualidade nas atividades desenvolvidas por vários profissionais. Cada vez mais esse posicionamento deve ser assumido pelos enfermeiros como forma de agregar e analisar as informações relevantes para a tomada de decisão e para o desempenho eficiente de todas as suas funções ${ }^{16}$.

Insta o desenvolvimento de mais pesquisas sobre a informatização no âmbito da enfermagem e, também, a formulaçãoe confecção de maisinstrumentos que possamaprimorar efadiltaro trabaho diário dos enfermeiros, usurruindo assim do crescenteavançotecnológico. Isso facilitaráaPBEem seuestagio final, atomada de decisão, oferecendo subsídios para a aplicaçãa dos resultados oriundos do campo prático de ondeemergiu oproblema. 
Comoevoluirdaspesquisasecomograndenúmerodeenfermeiros que resolveram se dedicar a esta área de atuação, temos um maior e melhor entendimento de informáticaem saúdeeem enfermagem ${ }^{18}$.

Este estudo está contido em um projeto maior que prevê o desenvolvimento de um banco de dados eletrônico com emissão de relatórios sobre o exame físico. Refere-se ao desenvolvimento de um sistema de informação para coleta de dados, tendo como base o exame físico em adultos e idosos, que proporcionará aos enfermeiros o registro de enfermagem de forma informatizada, eficiente e rápida.

0 desenvolvimento do referido projeto, incluindo a realização deste estudo, vem apontando para a necessidade de se propor a construção de um softwareparaaperfeiçoar asistematização da assistência de enfermagem em áreas hospitalares, mais precisamente em clínicas médicas. Entende-se que o hardwaree o software são meros meios para capturar, transferir, transformar o dado em informação com o objetivo de facilitar o desempenho, auxiliando a promover o melhor cuidado possível ao paciente/diente. ${ }^{18}$ Com isso espera-se contribuir à operacionalização da prática assistencial de enfermagem e para o futuro da profissão.

\section{CONCLUSÃO}

Conclui-se que o objetivo do estudo foi atingido e que a versão do instrumento elaborada com o presente estudo pode ser testada com os acadêmicos de enfermagem. Essa proposta do instrumento pode ser encarada como um meio facilitador para os acadêmicos de enfermagem, facilitando o registro das informações obtidas no exame físico de adultos e idosos hospitalizados, com a recomendação de ser testada também por enfermeiros que atuam nesses contextos.

\section{REFERÊNCIAS}

1. BanthaHD, LuceBR. Health care technology and its assessment: an international perspective. London: Oxford University; 1993.

2. Merhy EE. 0 ato de cuidar: a alma dos serviços de saúde. In: Ministério da Saúde (BR). Secretaria de Gestão do Trabalho e da Educação na Saúde. Departamento de Gestão da Educação na Saúde. Projeto Piloto da VER-SUSBrasil: cadernos de textos. Brasilia(DF); 2004, p.108-137. (Série B. Textos Básicos de Saúde). Disponível em: http://uwww.nescon.medicina.ufmg.br/ biblioteca/imagem/2103.pdf

3. Amante LN, et al. Ainterface entre o ensino do processo de enfermageme sua aplicação na prática assistencial. Rev Eletr Enferm. [on-line]. 2010; 12(1): 201-07. Disponível em: http://umw.fen.ufg.br/revista/v12/n1/pdf/v12n1a25.pdf

4. Fuly PSC. Ressignificando o gerenciamento da qualidade e a valorização do cuidado profissional: implantação e implementação da sistematização da assistência de enfermagem [tese]. Rio de Janeiro: Escola de Enfermagem Anna Nery, Universidade Federal do Rio de Janeiro; 2009

5. Conselho Federal deEnfermagem. Resolução COFENN³58/2009. Dispõesobre asistematização da assistência de enfermagem eaimplementação do processo de enfermagememambientes, públicos ou privados, em queocorreocuidadoprofissional deenfermagem, edáoutrasprovidências. Disponivelem:http://www.portalcofen.gov.br /2007/materias.asp?ArticlelD=10113\&sectionlD=34.
6. Matsuda LM, et al. Anotações/registro de enfermagem: instrumento de comunicação para a qualidade do cuidado? Rev Eletr Enferm. [on-line]. 2006; 8(03): 415-21. Disponível em: http://www.fen.ufg.br/revista/ revista8_3/v8n3a12.htm.

7. Évora YDM. A enfermagem na era da informática. Rev Eletr Enferm. [on-line] 2007; 09(01): 14. Disponível em: http://www.fen.ufg.br/revista/ v9/n1/v9n1a01_pt.htm.

8. Drummond JP, Silva E, Coutinho M. Medicina baseada em evidências: novo paradigma assistencial e pedagógico. $2^{\mathrm{a}}$ ed. São Paulo: Atheneu; 2002.

9. Cruz DALM, Pimenta, CAM. Prática baseada em evidências, aplicada ao raciocínio diagnóstico. Rev Latino-Am Enfermagem. [on-line]. 2005 maio/ junho: 13(03): 415-22. Disponível em: http://www.scielo.br/pdf/rlae/ v13n3/v13n3a17.pdf.

10. Marin HF. CIPE-Classificação Internacional para a Prática de Enfermagem. Versão 1. São Paulo: Algol Ed; 2007

11. Nóbrega MML, Garcia TR. Perspectivas de incorporação da Classificação Internacional para a Prática de Enfermagem-CIPE no Brasil. RevBras Enferm. [on-line]. 2005 mar/abr; 58( 2): 227-30. Disponível em:http://wnw.scielo.br/ scielo.php?pid=S0034-1672005000200020\&script=sci_arttext.

12. Lima LR, et al. Proposta de instrumento para coleta de dados de enfermagem em uma Unidade de Terapia Intensiva fundamentado em Horta. Rev Eletr Enferm. [on-line]. 2006; 8 (03): 349-57. Disponível em: http://www.fen.ufg.br/revista/revista8_3/v8n3a05.htm.

13. Garcia TR, Nóbrega MML. Processo de enfermagem: da teoria à prática assistencial e de pesquisa. Esc Anna Nery. 2009 jan/mar; 13 (1): 188-93.

14. Potter PA, Perry AG. Fundamentos de enfermagem, conceitos, processo e prática. $6^{a}$ ed. Rio de Janeiro: Guanabara Koogan; 2005.

15. Barros ALBL, et al. Anamnese e exame físico: avaliação diagnóstica de enfermagem no adulto. $2^{\mathrm{a}}$ ed. Porto Alegre: Artmed; 2010.

16. Sperandio DJ, Évora YDM. Planejamento da assistência de enfermagem: proposta de um software-protótipo. Rev Latino-Am Enfermagem. [on-line]. 2005 nov/dez; 13(6): 937-43. Disponível em: http://www.scielo.br/pdf/rlae/ v13n6/v13n6a04.pdf.

17. FulyPSC, et al. Correntes de pensamento nacionais sobre sistematização da assistência de enfermagem. RevBras Enferm. 2008 nov/dez; 61 (6): 883-87.

18. Marin C, Olm ICK. Perspectivas atuais da informática em enfermagem. Rev Bras Enferm. [on-line]. 2006 maio/jun; 59(3): 354-55. Disponívelem:http://wnw.scielo.br/ scielo.php?script=sci_arttext\&pid=S0034-71672006000300019. 

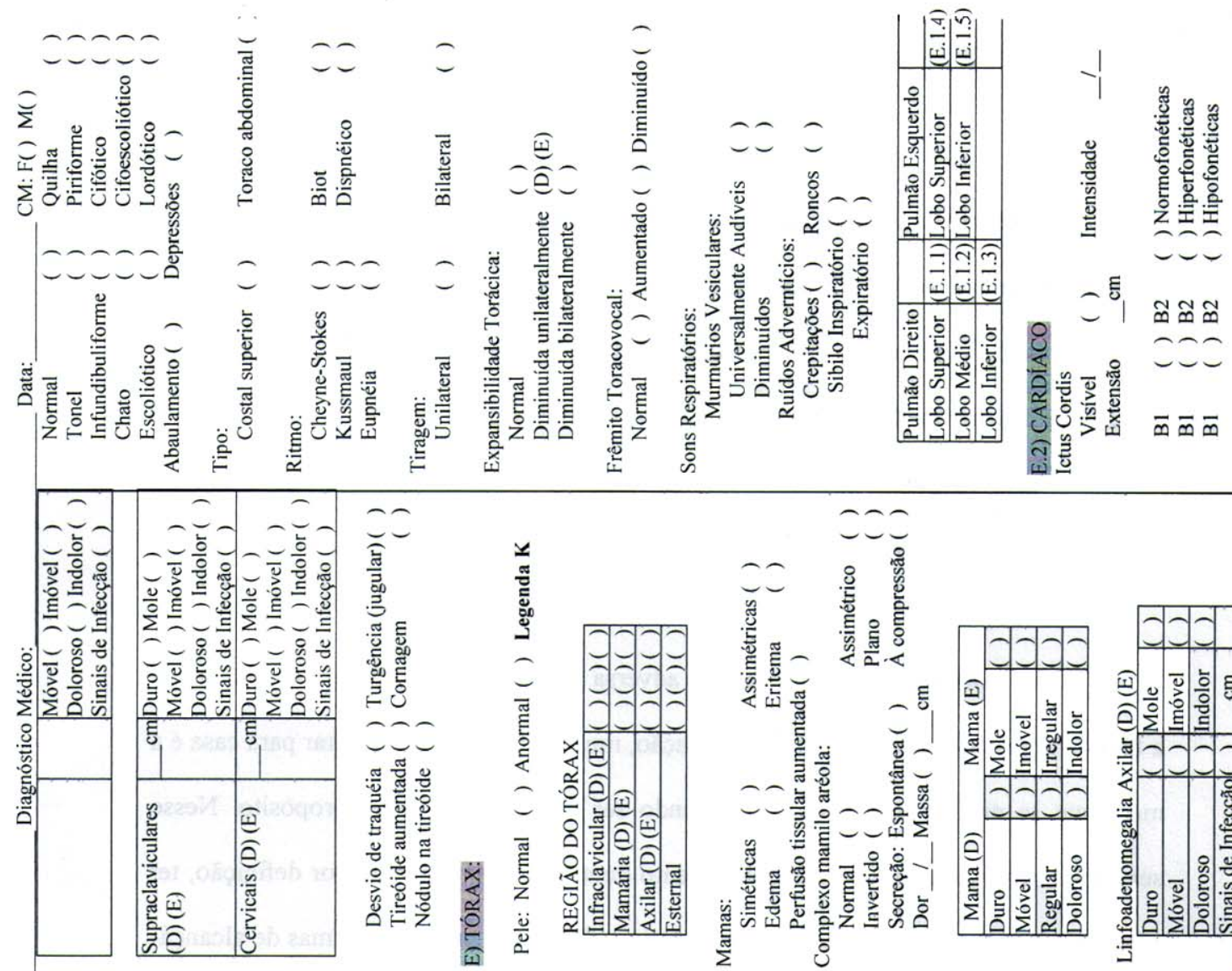

Wi

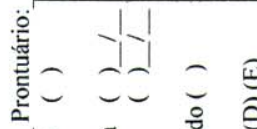

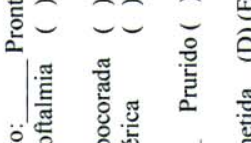

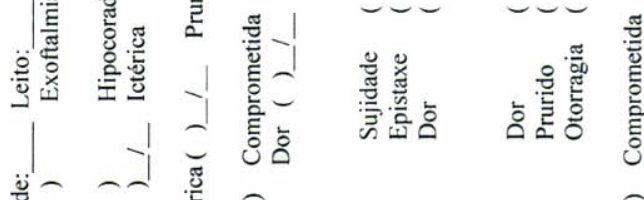

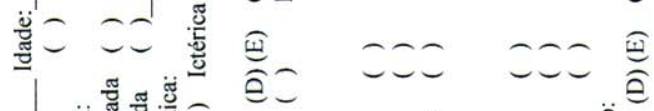

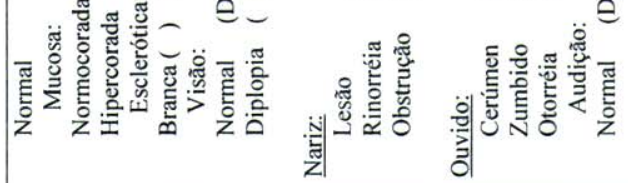
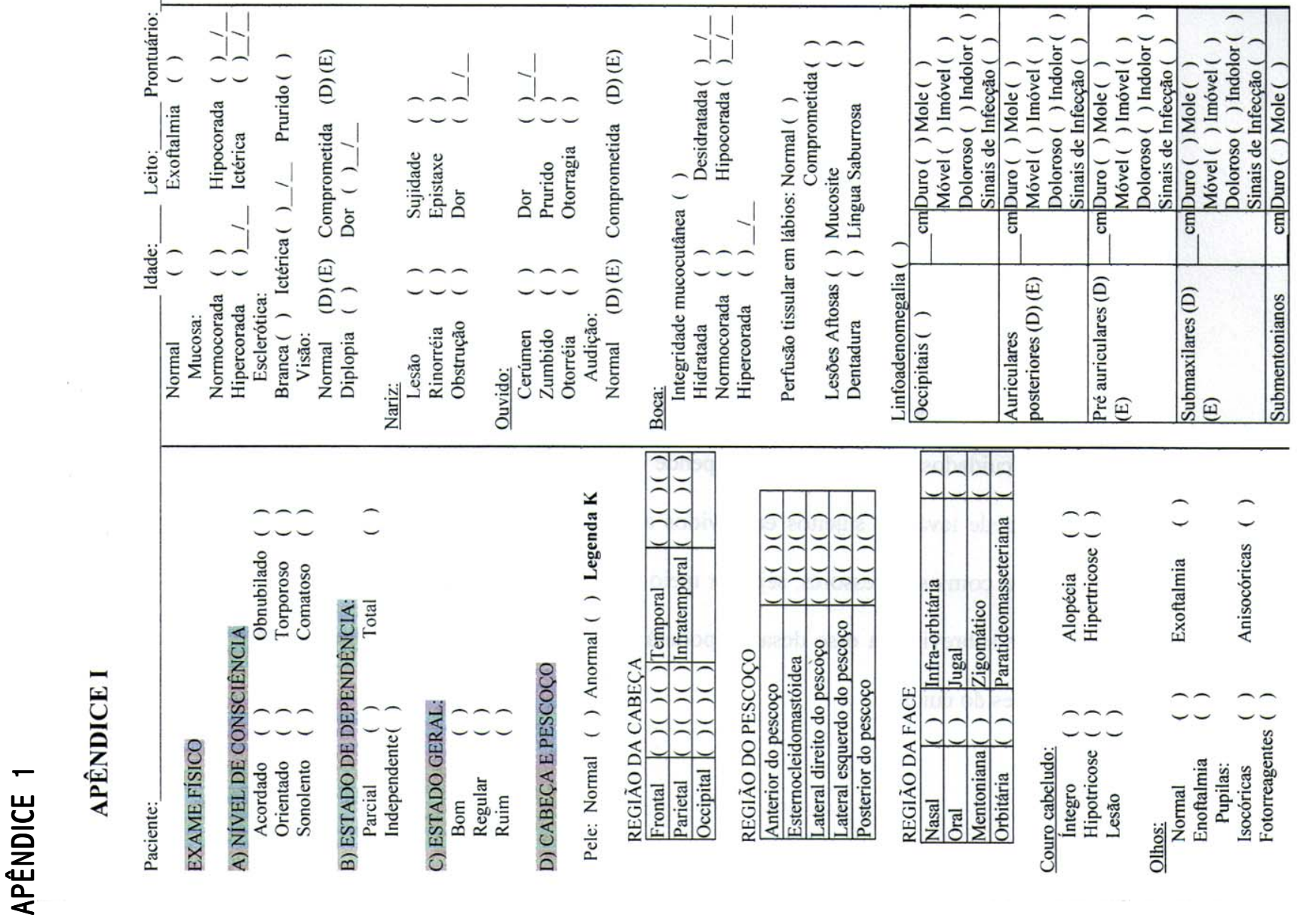

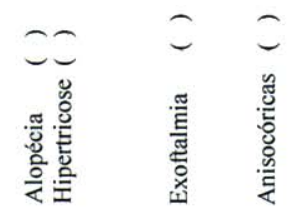

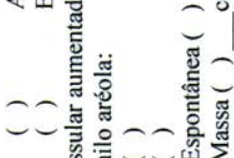

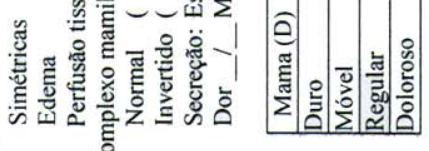
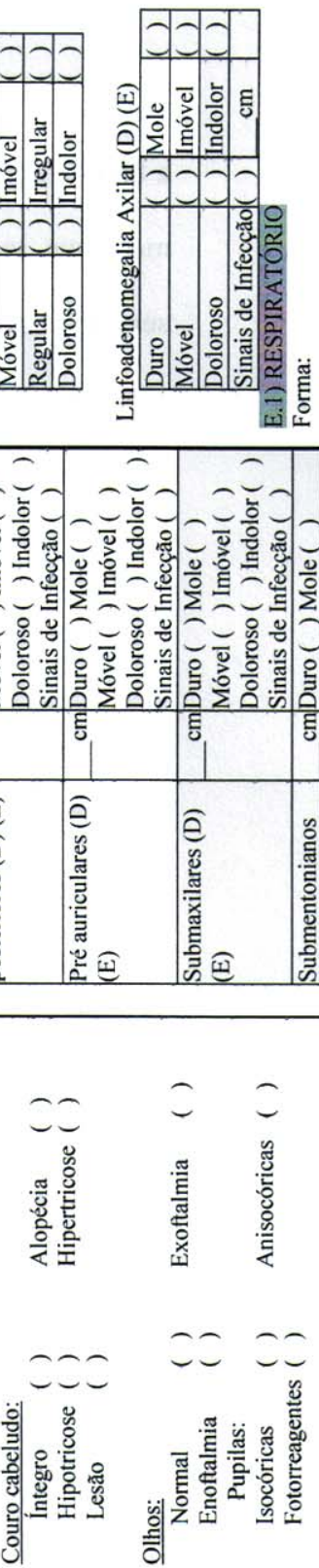

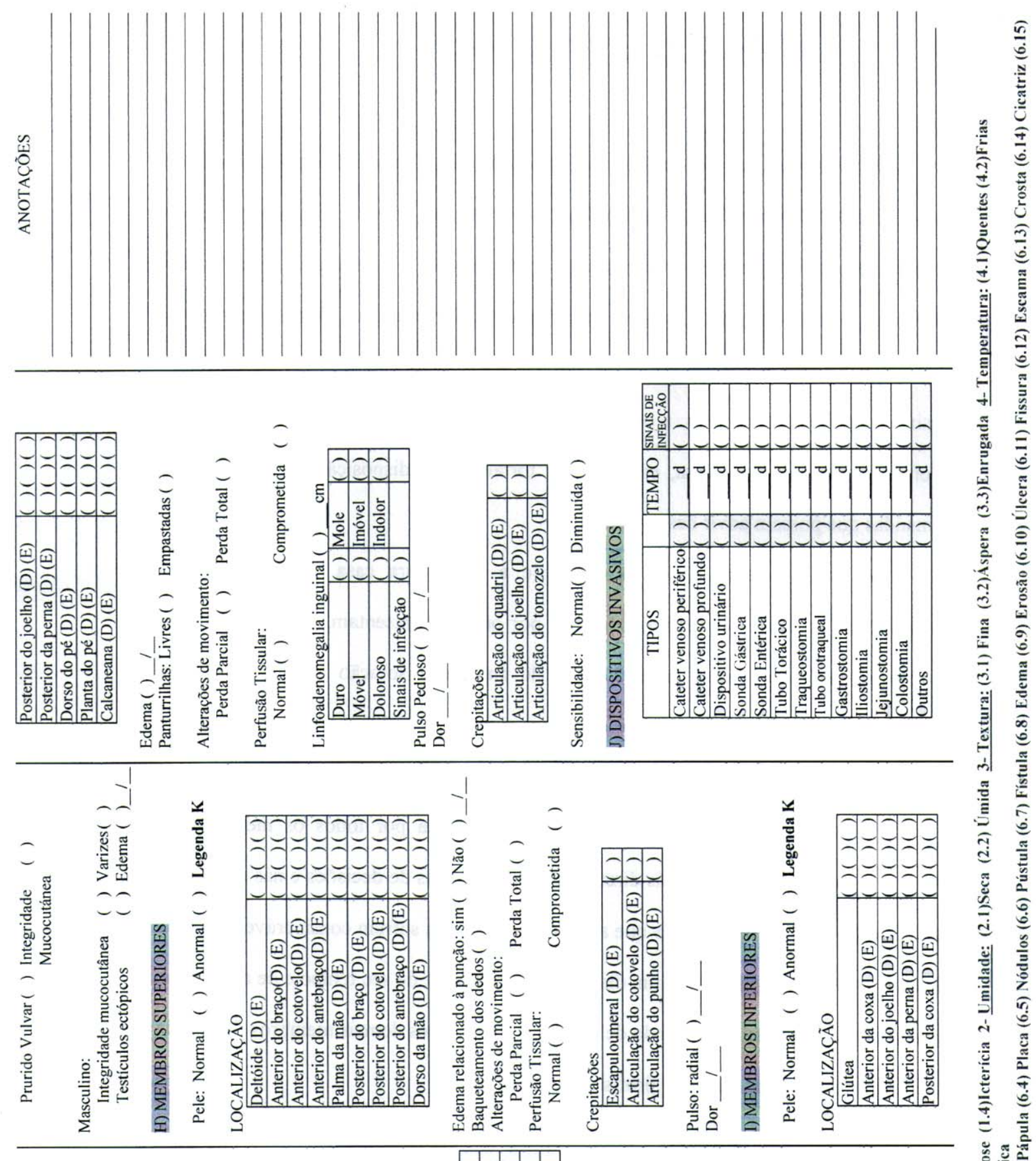

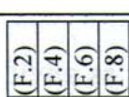
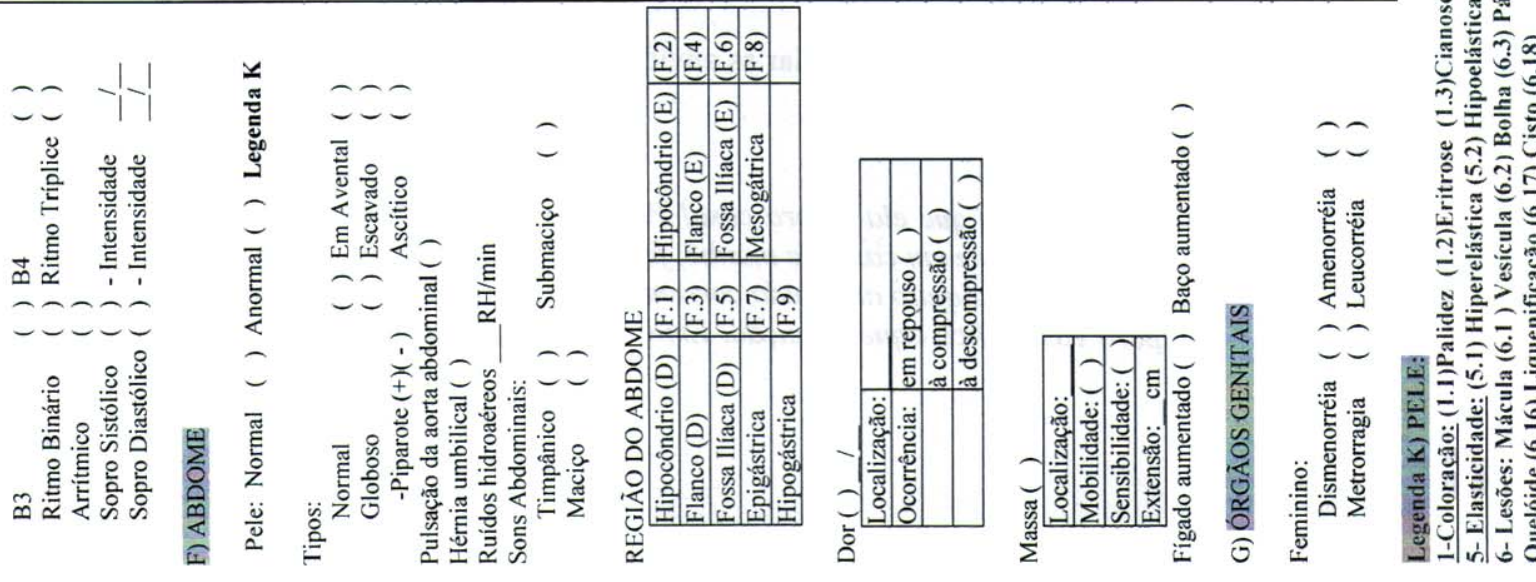